\title{
Long-Term Administration and Outcomes of Tolvaptan for Hepatic Edema
}

Takuya Iwamoto*, Masaki Maeda, Issei Saeki, Isao Hidaka, Tsuyoshi Ishikawa, Taro Takami, and Isao Sakaida

Department of Gastroenterology and Hepatology, Yamaguchi University Graduate School of Medicine, 1-1-1 Minami-Kogushi, Ube-Yamaguchi 755-8505, Japan

*Corresponding author: Takuya Iwamoto, Department of Gastroenterology and Hepatology, Yamaguchi University Graduate School of Medicine, 1-1-1 Minami-Kogushi, Ube-Yamaguchi 755-8505, Japan, Tel: +81-836-22-2241; E-mail: t_iwamot@yamaguchi-u.ac.jp

Received date: August 11, 2017; Accepted date: August 23, 2017; Published date: August 25, 2017

Copyright: $\odot 2017$ Iwamoto T, et al. This is an open-access article distributed under the terms of the Creative Commons Attribution License, which permits unrestricted use, distribution, and reproduction in any medium, provided the original author and source are credited.

\section{Abstract}

Objective: Tolvaptan is an oral vasopressin V2 receptor antagonist that became available as therapy for decompensated hepatic cirrhosis-induced ascites in 2013. It has now been more than 3 years since hepatic edema was included in the indication. We investigated use of tolvaptan in our department, including long-term administration, discontinuation, and re-administration after discontinuation.

Methods: The subjects were 62 patients with hepatic edema treated with tolvaptan between september 2013 and december 2016. Physical parameters and blood data during hospitalization and the course thereafter were investigated retrospectively.

Results: The median age was 71.2 (49-87) years old, the mean Child-Pugh score was $9.5 \pm 1.7$, background liver Hepatitis C virus /Hepatitis B virus/Alcohol/Non-alcoholic steatohepatitis/Others=38/5/6/5/8, and 41 patients were complicated by hepatocellular carcinoma. Tolvaptan was initiated at $3.75 \mathrm{mg}$ in all patients, and the dose was increased to $7.5 \mathrm{mg}$ if the effect was insufficient after administration for 3 days. Patients who lost $\geq 1.5 \mathrm{~kg}$ weight after tolvaptan administration for one week were defined as early responders (39/62,62.9\%). The median duration of tolvaptan administration was 96 (7-992) days. Tolvaptan was continued in 46 patients at the outpatient clinic. In 5 patients, tolvaptan was discontinued because ascites improved, but 3 required readministration. Of the 46 patients who received continuous tolvaptan, 18 died, but 14 did not require removal of ascites by puncture or Cell-free and Concentrated Ascites Reinfusion Therapy before death. In an analysis of outcomes by Log-rank test, there was no significant relationship with Child-Pugh score or Model for End-Stage Liver Disease score, but significant effects of hepatocellular carcinoma and continuous tolvaptan. In multivariate analysis using Cox proportional hazards regression analysis, hepatocellular carcinoma (hazard ratio 3.366) and continuous tolvaptan (hazard ratio 7.291) were identified as significant independent factors related to outcome.

Conclusion: Continuous administration of tolvaptan may enable long-term control of hepatic edema and improve the outcome of patients with hepatic cirrhosis.

Keywords Decompensated hepatic cirrhosis; Hepatic edema; Tolvaptan; Vasopressin; Ascites; Hepatocellular carcinoma; Continuous administration

\section{Introduction}

Hepatic cirrhosis is a progressive disease that reaches the decompensated stage at an annual rate of 5-7\%. Diverse complications, such as hepatic encephalopathy and gastroesophageal varices, develop in decompensated cirrhosis, and ascites reduces quality of life (QOL) and strongly influences the life prognosis of patients with hepatic cirrhosis [1]. Aldosterone antagonists and loop diuretics have been used for many years as drug therapy for decompensated hepatic cirrhosis-induced ascites [2], but treatment with these diuretics does not have a sufficient effect in some patients, and electrolyte imbalance and renal impairment with diuretic dose escalation are problematic [3].

In 2013, tolvaptan an oral vasopressin V2 receptor antagonist became available for treatment of fluid retention associated with hepatic cirrhosis. The mechanism of action of tolvaptan differs from those of conventional diuretics [4]. Tolvaptan is an aquaretic that induces removal of excess water without electrolyte excretion, so that the effect is not influenced by the blood albumin level, unlike other diuretics [5]. We previously showed that tolvaptan is likely to be effective in patients with favourable renal function [6] and several studies have reported a similar finding [7,8], but long-term administration was investigated in only a few studies. More than 3 years have passed since hepatic edema was included in the indication of tolvaptan, and findings in actual medical practice are now emerging, along with the results of clinical trials $[9,10]$. In this study, we investigated current use of tolvaptan in our department, including long-term administration, discontinuation, and re-administration after discontinuation.

\section{Subjects and Methods}

The subjects were 69 patients with hepatic edema who were treated with tolvaptan because of difficulty with ascites control with existing diuretic treatment between september 2013 and december 2016. After excluding patients in whom body weight was unmeasurable and those with missing data, 62 patients were evaluated in the study. Baseline clinical and biochemical data of patients were recorded at admission. The Child-Pugh score and the Model for End-Stage Liver Disease (MELD) score were calculated from the baseline data. 


\begin{tabular}{|l|l|}
\hline Variable & Value \\
\hline Age (years) & $71.2(49-87)$ \\
\hline Sex (male) & $35(56 \%)$ \\
\hline Body weight (kg) & $59.1 \pm 12.4$ \\
\hline Height (cm) & $157.4 \pm 8.9$ \\
\hline Etiology (HCV/HBV/Alcohol/NASH/Others) & $38 / 5 / 6 / 5 / 8$ \\
\hline Child class (A/B/C) & $1 / 29 / 32$ \\
\hline Child-Pugh score & $9.5 \pm 1.7$ \\
\hline MELD score & $12.9 \pm 3.7$ \\
\hline Serum albumin (g/dL) & $2.54 \pm 0.42$ \\
\hline Serum creatinine (mg/dL) & $1.18 \pm 0.44$ \\
\hline Serum sodium (mEq/L) & $136.3 \pm 4.6$ \\
\hline Loop diuretic dose (mg) & $41.6 \pm 28.0$ \\
\hline Spironolactone dose (mg) & $37.7 \pm 16.3$ \\
\hline HCC (with/without) & $41 / 22$ \\
\hline HCC stage (I/l//II//IV) & $4 / 5 / 13 / 19$ \\
\hline Vp (O/1/2/3/4) & $26 / 2 / 4 / 4 / 5$ \\
\hline Data are shown as median (range), number (\%) or mean \pm SD. \\
\hline
\end{tabular}

ingestion. No other serious complication occurred. Tolvaptan was continued thereafter in 46 patients at the outpatient clinic, and the median duration of administration was 191 (14-992) days. The course after tolvaptan administration is shown in Figure 1.

Table 1: Baseline characteristics of the patients.

We assessed tumor stage according to the criteria of the Liver Cancer Study Group of Japan, 6th edition [11]. All serum samples were obtained from peripheral vein at early morning after fasting and resting in a supine position. Tolvaptan was initiated at a dose of 3.75 $\mathrm{mg}$ in all patients, and the dose was increased to $7.5 \mathrm{mg}$ if the effect was insufficient after administration for 3 days. The dose was increased to $7.5 \mathrm{mg}$ in 33 patients (53.2\%). Patients whose body weight decreased by $\geq 1.5 \mathrm{~kg}$ after tolvaptan administration for one week were defined as early responders [12]. The median duration of tolvaptan treatment in the 62 patients was 96 (7-992) days. Details of the patients are shown in Table 1 . The study was performed in conformity with the regulations of the institutional ethics committee (approval number: H27-096).

Height, body weight, and blood and urinalysis data were collected and are presented as median (range), number (\%) or mean \pm standard deviation (SD). Statistical analysis was conducted using JMP9 (SAS Institute, Cary, NC, USA). A Chi-square test or Fisher exact test was used to evaluate differences between two groups. Changes from baseline in data in the same group were evaluated by t-test. Missing data were excluded from analyses. Kaplan-Meier survival curves were evaluated by log-rank test. A cox proportional hazard model was used to perform multivariate analysis. All tests were two-tailed and $\mathrm{P}<0.05$ was taken to indicate statistical significance.

\section{Results}

The response rate to tolvaptan was $62.9 \%$ (39/62 patients). During the tolvaptan administration period, 5 patients complained of thirst and 3 patients had dry skin. Both symptoms may have been complications due to dehydration and both were improved by water

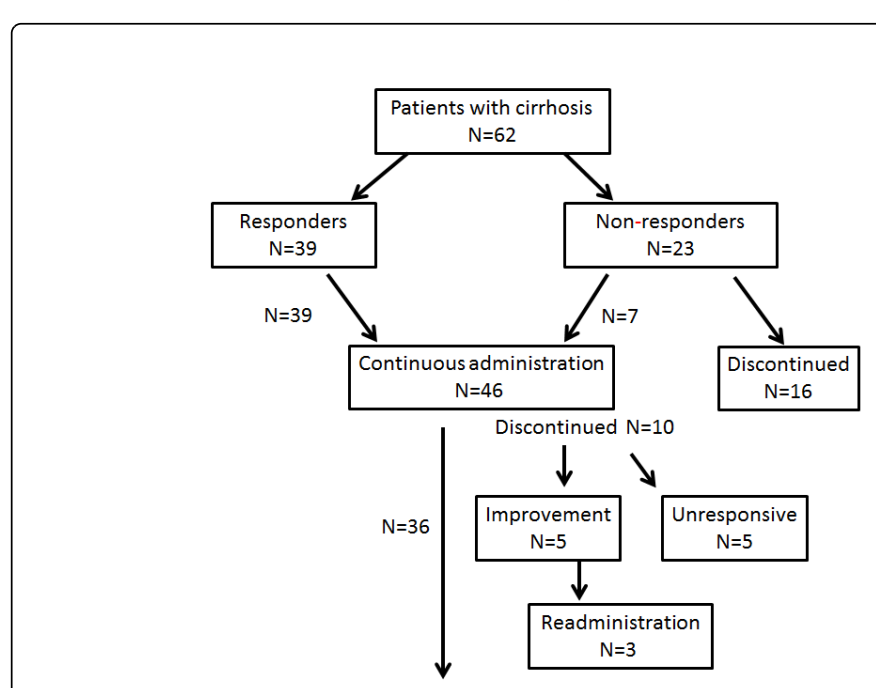

Figure 1: Therapeutic effects of tolvaptan in the 62 patients in the study. The course after tolvaptan administration is shown.

Ascites did not aggravate throughout the observation period in $73 \%$ of the patients, and the mean aggravation-free period was $324 \pm 282$ days (Figure 2). Tolvaptan was discontinued at the outpatient clinic in 10 patients: 5 became refractory to tolvaptan (median administration period: 30 (20-38) days) and ascites was improved in the other 5 patients (median administration period: 35 (14-56) days).

Readministration was required in 3 of the 5 patients in whom tolvaptan was withdrawn because ascites improved (median time to readministration: 25 (14-57) days). Of the other 2 patients, one died of other diseases one month after withdrawal of tolvaptan, and tolvaptan withdrawal was continued in the other patient, in whom liver function was improved by continuous abstinence from alcohol intake (Figure 1).

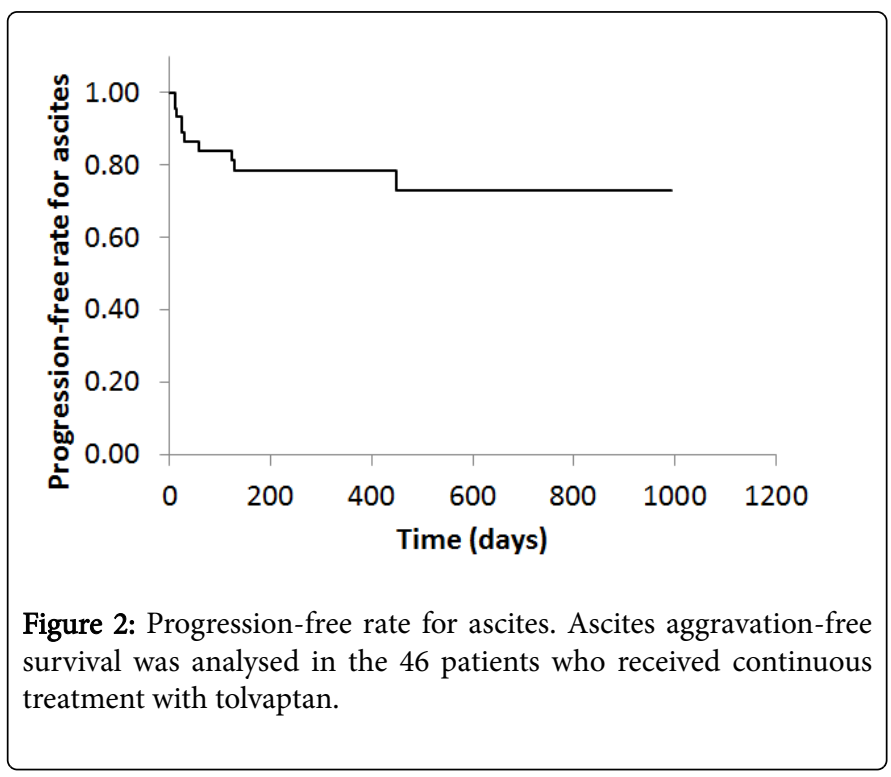


Page 3 of 4
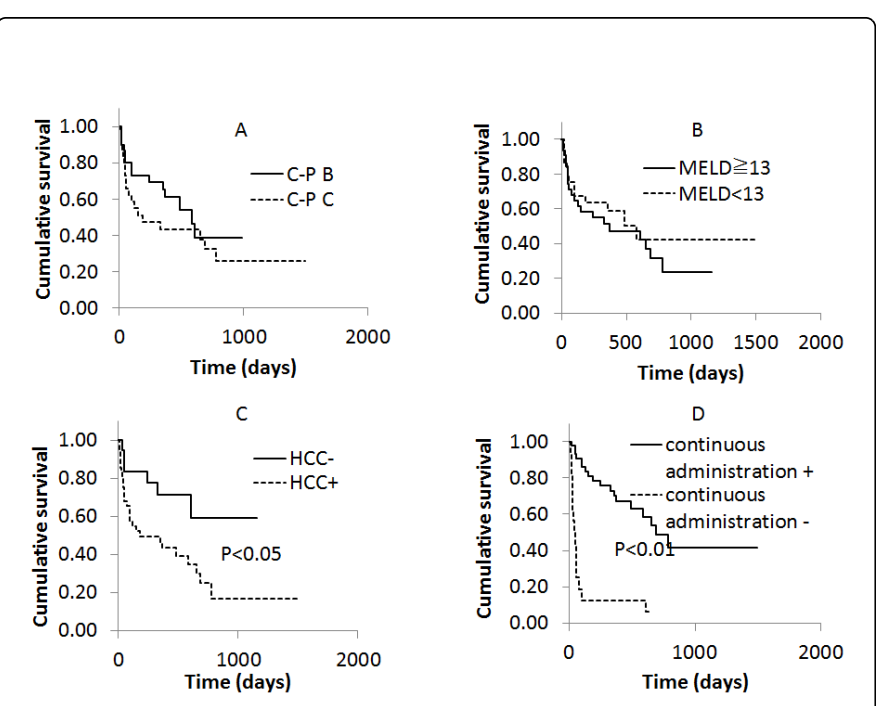

Figure 3: Kaplan-Meier analysis of patients treated with tolvaptan. A: Patients in Child-Pugh classes B (solid lines) and C (dotted lines). B: Patients with MELD scores $\geq 13$ (solid lines) and $<13$ (dotted lines). C: Patients with (dotted lines) and without (solid lines) HCC complication. D: Patients treated with (solid lines) and without (dotted lines) continuous tolvaptan administration. Analysis by Log-rank test (Cochran-Mantel-Haenszel).

Eighteen of the 46 patients continuously treated with tolvaptan died, with causes of death of hepatocellular carcinoma $(n=9)$, liver failure $(n=6)$, and other diseases $(n=3)$. In 14 of these patients, ascites was controlled until death by continuation of oral tolvaptan alone, without ascites removal by puncture and Concentrated Ascites ReinfusionTherapy (CART).

An analysis of factors influencing survival by log-rank test showed no significant relationship with the Child-Pugh class or MELD score, but significant effects of hepatocellular carcinoma and continuous tolvaptan administration (Figure 3). In multivariate analysis using a Cox proportional hazards regression model, hepatocellular carcinoma (hazard ratio 2.973) and continuous tolvaptan (hazard ratio 7.307) were identified as significant independent factors related to outcome (Table 2).

\begin{tabular}{|c|c|c|c|}
\hline Factor & Hazard ratio & $\mathbf{9 5 \%} \mathbf{C l}$ & P-value \\
\hline MELD score $(\geq 13)$ & 1.312 & $0.542-3.175$ & 0.547 \\
\hline Child-Pugh class (B or C) & 1.441 & $0.621-3.347$ & 0.395 \\
\hline Hepatocellular carcinoma & 3.366 & $1.290-8.783$ & 0.013 \\
\hline $\begin{array}{c}\text { Continuous administration of } \\
\text { tolvaptan }\end{array}$ & 7.291 & $3.313-16.04$ & 0.000 \\
\hline
\end{tabular}

Table 2: Results of multivariate analysis of factors associated with outcomes of treatment of ascites using tolvaptan determined using a cox proportional hazards model.

\section{Discussion}

Three years have passed since tolvaptan became administrable at clinical sites, and consequently the number of patients treated with this drug for a prolonged period has increased. In the current study, tolvaptan was effective for hepatic edema resistant to conventional diuretics and caused no serious adverse effects, which suggests that this drug can be continuously administered safely. Aggravation of ascites was prevented by continuous tolvaptan in $73 \%$ of patients with hepatic cirrhosis and these patients were able to receive treatment at the outpatient clinic. However, re-administration was necessary for $60 \%$ of patients in whom tolvaptan was withdrawn because ascites improved. Reduction of activities of daily living (ADL) and QOL by retention of ascites was prevented until death in patients who received continuous tolvaptan. This suggests that long-term administration of tolvaptan is beneficial, and multivariate analysis suggested that continuous tolvaptan improved outcomes in patients with hepatic cirrhosis. There is a concern with regard to medical costs, and it has been reported that long-term administration of tolvaptan does not influence the prognosis of heart failure [13].

The Child-Pugh class and MELD score, which reflect hepatic functional reserve [14], were not significantly related to outcome, whereas ascites and hyponatremia have been found to be strong risk factors for death in a population with a relatively low MELD score [15]. However, the present study comprised patients with hepatic cirrhosis with intractable ascites, and ascites control may more strongly influence the outcome, rather than hepatic functional reserve, in such a population. Tolvaptan administration could be temporarily discontinued because of elimination of ascites in only one patient whose liver function was improved by abstinence from alcohol, suggesting that continuous administration is appropriate for cases with other causes of disease. Confirmation of this conclusion will require investigation of long-term administration of tolvaptan in a prospective study.

In conclusion, continuous administration of tolvaptan may enable long-term control of hepatic edema and improve the outcome of patients with hepatic cirrhosis.

\section{Acknowledgment}

This work was supported by JSPS KAKENHI Grant Number $17 \mathrm{~K} 15949$.

\section{References}

1. D'Amico G, Garcia-Tsao G, Pagliaro L (2006) Natural history and prognostic indicators of survival in cirrhosis: a systematic review of 118 studies. J Hepatol 44: 217-231.

2. Runyon BA (1997) Treatment of patients with cirrhosis and ascites. Semin Liver Dis 17: 163-173.

3. Moore KP, Wong F, Gines P, Bernardi M, Ochs A, et al. (2003) The management of ascites in cirrhosis: report on the consensus conference of the International Ascites Club. Hepatology 38: 258-266.

4. Yamamura Y, Nakamura S, Itoh S, Hirano T, Onogawa T, et al. (1998) OPC-41061, A highly potent human vasopressin V2-receptor antagonist: pharmacological profile and aquaretic effect by single and multiple oral dosing in rats. J Pharmacol Exp Ther 287: 860-867.

5. Sakaida I, Nakajima K, Okita K, Hori M, Izumi T, et al. (2015) Can serum albumin level affect the pharmacological action of tolvaptan in patients with liver cirrhosis? A post hoc analysis of previous clinical trials in Japan. J Gastroenterol 50: 1047-1053.

6. Iwamoto T, Maeda M, Hisanaga T, Saeki I, Fujisawa K, et al. (2016) Predictors of the effect of tolvaptan on the prognosis of cirrhosis. Intern Med 55: 2911-2916. 
Citation: Iwamoto T, Maeda M, Saeki I, Hidaka I, Ishikawa T, et al. (2017) Long-Term Administration and Outcomes of Tolvaptan for Hepatic Edema. J Liver 6: 220. doi:10.4172/2167-0889.1000220

Page 4 of 4

7. Kawaratani H, Fukui H, Moriya K, Noguchi R, Namisaki T, et al. (2017) Predictive parameter of tolvaptan effectiveness in cirrhotic ascites. Hepatol Res 47: 854-861.

8. Nakanishi H, Kurosaki M, Hosokawa T, Takahashi Y, Itakura J, et al. (2016) Urinary excretion of the water channel aquaporin 2 correlated with the pharmacological effect of tolvaptan in cirrhotic patients with ascites. J Gastroenterol 51: 620-627.

9. Okita K, Sakaida I, Okada M, Kaneko A, Chayama K, et al. (2010) A multicenter, open-label, dose-ranging study to exploratively evaluate the efficacy, safety, and dose-response of tolvaptan in patients with decompensated liver cirrhosis. J Gastroenterol 45: 979-987.

10. Sakaida I, Kawazoe S, Kajimura K, Saito T, Okuse C, et al. (2014) Tolvaptan for improvement of hepatic edema: A phase 3, multicenter randomized, double-blind, placebo-controlled trial. Hepatol Res 44: 73-82.

11. Liver Cancer Study Group of Japan (2015) The general rules for the clinical and pathological study of primary liver cancer, 6th edn. : TokyoKanehara \& Co., Ltd.
12. Hiramine $\mathrm{Y}$, Uojima $\mathrm{H}$, Nakanishi $\mathrm{H}$, Hiramatsu A, Iwamoto $\mathrm{T}$, et al. (2017) Response criteria of tolvaptan for the treatment of hepatic edema. J Gastroenterol [ Epub ahead of print].

13. Konstam MA, Gheorghiade M, Burnett JC Jr, Grinfeld L, Maggioni AP, et al. (2007) Effects of oral tolvaptan in patients hospitalized for worsening heart failure: the EVEREST Outcome Trial. JAMA 297: 1319-1331.

14. Huo TI, Wu JC, Lin HC, Lee FY, Hou MC, et al. (2005) Evaluation of the increase in model for end-stage liver disease (DeltaMELD) score over time as a prognostic predictor in patients with advanced cirrhosis: risk factor analysis and comparison with initial MELD and Child-TurcottePugh score. J Hepatol 42: 826-832.

15. Heuman DM, Abou-Assi SG, Habib A, Williams LM, Stravitz RT, et al. (2004) Persistent ascites and low serum sodium identify patients with cirrhosis and low MELD scores who are at high risk for early death. Hepatology 40: 802-810. 